\title{
Modeling of Evolution and Sustainability of Rational Trust in Dynamic VOs
}

\author{
Morice Daudi and Simon Samwel Msanjila \\ Faculty of Science and Technology, Mzumbe University, Mzumbe, Tanzania \\ $\{$ dmorice, smsanjila\} @mzumbe.ac.tz
}

\begin{abstract}
Modeling of trust sustainability and evolution among partners of a dynamic Virtual Organizations (VO) whose collaboration is already established, poses high challenge to such virtual co-working environment. Research in VOs has not established suitable modeling approaches for analyzing evolution and sustainability of rational trust and related assessment mechanisms for the collaboration which is already in progress. This is very necessary as it has now been proved that the base trust level acquired by VO partners during the VO formation is not static and cannot to remain constant over entire period of collaborative business execution. Thus proper modeling and analysis approaches for measuring the continuous changing of trustworthiness of organization need to be developed. This paper proposes approaches and mechanisms for modeling and assessment mechanism for supporting interorganizational trust sustainability and evolution.
\end{abstract}

Keywords: trust evolution, trust sustainability, VO, trust modeling.

\section{Introduction}

In traditional business undertakings, organizations tend to struggle and win business opportunity individually. Nowadays, SME organizations in particular are less and less capable to afford acquiring and executing business opportunities individually, and now a new form of working in cooperation networks is being adopted (Msanjila and Afsarmanesh, 2007). The emerging approach which is now being adopted by collaborating organizations is Virtual Organizations (VO) which are configured constituting organization that are members of a long-term strategic alliance. Dynamic VOs which are mostly preferred are those temporary consortiums whose structure and particularly partners may change during the collaboration. The VOs characteristics include tendency of organizations to create "virtual size" by becoming partners but at the same time trying to preserve the flexibility of small companies (Riemer et al., 2001); and there is no leading or subordinate" (Appel \& Behr, 1996).

Smooth operation of the VO needs to be supported with rational trust among the partners to provide a bonding factor and a glue to stick these partners together. Established inter-organizational trust through rational mechanisms is used to enhance partner's confidence that a specific member of the VO can execute its assigned roles appropriately to collaboratively achieve the common goal(s). Despite the different and opposing perception of trust, we perceive trust in $\mathrm{VO}$, which only rational trust is 
suitable to apply, as the objective-specific confidence of a trustor in a trustee, based on the results of rational (fact-based) assessment of trust level of the trustee (Msanjila, 2009). The main input into trust analysis is measurable organization's performance.

Organizations collaborate in the VO for a number of reasons including saving time and decreasing development process, sharing costs and risks with partners, improving resource utilization, and gain access to new markets through partnership (Nami and Malekpour, 2008). Although, it is clear regarding its urgency, trust of the VO partner does not remain static, but varies depending on many influencing factors including comparative nature. Since the level of trust for an organization is dynamic and evolves over time, then comprehensive approaches for managing rational trust in VOs whose collaboration is already in progress is important. It is because, with a rising number of relationships and especially short-time relationships, as needed in VOs, conventional approach to build trust among business partners has proven to be inefficient (Wehmeyer and Riemer, 2007). Scientific mechanisms for modeling trust sustainability and supporting for a VO evolution are needed so that VOs remain bonded to achieve their goals and undergo evolution when required.

This paper describes classification of trust (section 2), presents approaches and mechanisms for modeling and assessment of rational trust (section 3), suitable for analysis of inter-organizational trust sustainability and evolution.

\section{Classification of Trust}

Trust can be classified in two aspects, namely; (1) Subjective trust, and (2) Objective trust. In the past, trust used to be perceived as subjective (opinion-based); posing difficulties in its evaluation and formal reasoning on analysis of its results, due to the use of opinion-based data, thus being risky as it is biased (Msanjila 2012). In subjective trust, a trustee is evaluated based on opinions, suggestions, and recommendations and sometimes polling. It is a biased trust and very difficult to reason about its results. Measurement and assessment of the subjective trust provides questionable results for a VBE/VO member to be trusted, because data sources are not properly characterized and are difficult to factually measure.

Objective trust is the results of the rational (fact-based) assessment of the trust level (Msanjila and Afsarmanesh, 2007). It is the trust whose assessment is based on organizational performance data (fact-based), and provides results which an individual can reason about. Trust in the past was measured statically and did not consider the variation of trustworthiness with time. Such dynamic behavior of trust level accounts for existence of Trust Sustainability and Trust Evolution. Trust sustainability is the process whereby a VO partner maintains their trustworthiness to the specific trust level to enable it remains acceptable in the VO during execution of the activities that lead into achieving the common goal of their collaboration (Msanjila, 2012). Each VO partner has to maintain its trust level above the acceptable value, namely the required specific trust level for the VO. If the trust level falls below the specific trust level of the VO then the said VO partner cannot be trusted and its capacity to execute its assigned roles shall be doubtable. When such an organization fails to sustain its level of trust, the VO undergoes evolution from which new VO partners are selected from VBE to fill the gap of departing partner with poor trust. 
When new partners are added in the VO naturally trust level of others will change because of the comparative analysis and measurement applied. This process is referred to as trust evolution. When new member(s) are added in a VO, there will be a change on an average trust level of the VO, which implies trust evolution.

\section{Trust Evolution Assessment Mechanism}

In research there is still lack of suitable mechanisms for the assessment of rational trust of collaborating VO partners. Mechanisms for assessing rational trust during the selection of VO partners measure the basic trust level. These mechanisms cannot be applied to assess trust in already existing consortiums, because trust level is not static but changes with time. Current mechanisms for assessing rational trust consider trust level at a specific point in time (static assessment). These mechanisms do not address the evolution of trust with time and thus assumes trustworthiness of organizations is static once measured. This has proven to be not the case and therefore mechanism for assessing evolving trust level of organizations are required as proposed in this paper.

The assessment mechanisms that can support trust sustainability and evolution among partners collaborating in VO over entire period of business execution as proposed in this paper are modeled based on performance acquired or achieved by the partners in the specific VO. The proposed models are developed applying statistics concepts that can capture variation measures with time. The statistics concepts, namely: (1) measure of central tendency (mean) and (2) dispersion (mean deviation and standard deviation) are applied to develop mechanisms to capture the evolution of trust in VO partners. In the subsequent subsection three (3) rational trust assessment mechanisms discussed in detail, and finally a best-to-fit model is proposed. In each presented model, the following assumptions are made: Base period: Is a specific interval time (monthly, quarterly, semi-annually, and annually) which is considered to be a period for assessment base trust level of VO partner. Base trust level: The trust level acquired by a VO partner at specific point in time.

\subsection{First-Level Model: Mean Estimation}

This approach considers mean estimation for trust level of VO partners in a given period (1). The mean trust level estimated at current period becomes the target score for which every member's trust level should not fall below the threshold in the next period. For example if the base period is assumed to be quarterly, then mean trust level is computed using trust level of individual partner. After a lapse of three (3) months, the new acquired trust level for each VO partner is compared against that mean trust level to find if it is less than, equal to or greater than mean trust level at such current period (refer figure 1 and figure 2). Mean trust level is calculated as

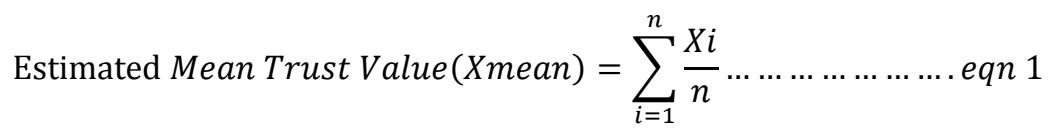

Where $\mathrm{Xi}$ is a trust level and $\mathrm{n}$ is the number of VO partners participating in the VO. In figure $1, \mathrm{M}$ stands for a VO partner while TL stands for Trust Level of the partner. 


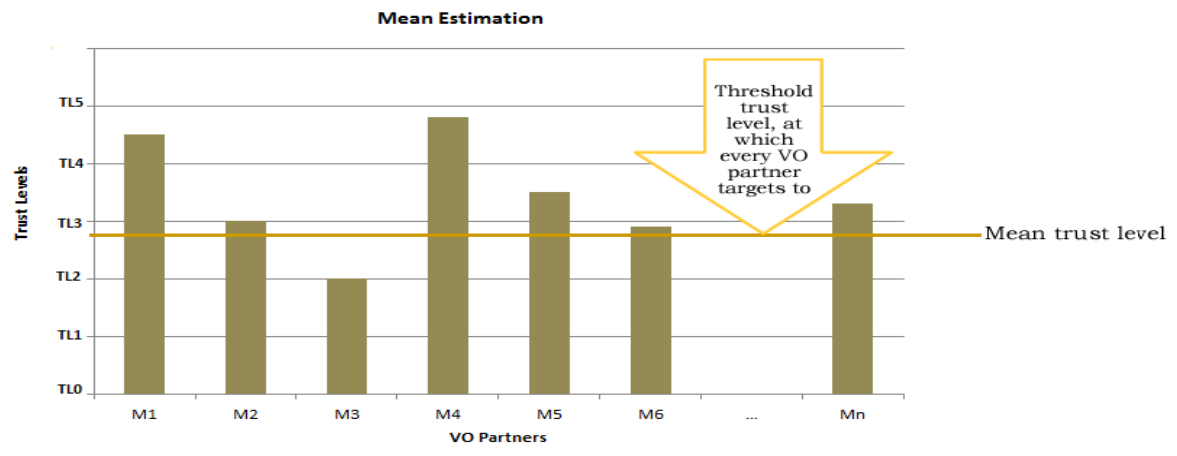

Fig. 1. Trust assessment by mean estimation method

It may happen that the mean trust level be above of some levels of trust of some VO partners at a given period (figure 1). In such situation: (1) Fair play to some of the partners $\left(\mathrm{M}_{3}\right)$ is ignored, but favoring partners whose trust values were high $\left(\mathrm{M}_{1}, \mathrm{M}_{2}\right.$, $\mathrm{M}_{4}, \mathrm{M}_{5}, \mathrm{M}_{6}$ ) during the base assessment period and (2) Assessment does not provide a target point for a particular partner, looks at a single point for all member to achieve.

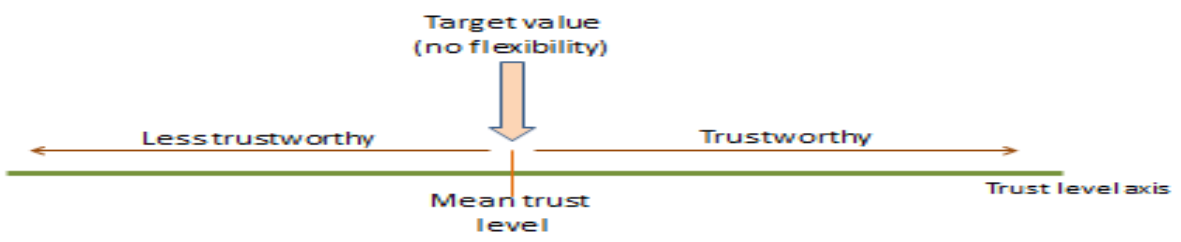

Fig. 2. Linear presentation of cut-off point in the statistical mean estimation method

The impact of this assessment includes: (1) Partners whose trust levels are below mean estimated trust value are deprived of a fair chance to build trust over others; (2) Partners whose trust level scores are above mean estimated level have greater confidence to build trust over others; (3) The model does not give a range for variation in trust level, rather aims at single point and (4) The mechanism has a narrow space for trust variation limits. This model is suitable for discovering partners whose levels of trust have fallen below specific VO's mean trust level for removing underperforming partners and replace them with other new partners (figure 2).

\subsection{Second-Level Model: Mean Deviation}

The mean deviation (average deviation) is the mean of the absolute deviations of observations from some suitable average which may be the arithmetic mean, the median or the mode. The difference (how far is each data from the average) between a specific data and the average itself, is called deviation. Considering arithmetic mean for this model, mean deviation is the average of how far is each individual data element from the mean. This difference is given by the following formula. 
If the negative sign is ignored, the deviation is written as

$$
\text { Mean }=\mid X-\text { Average } \mid
$$

and is read as mod deviation. For ungrouped data, mean deviation is given by

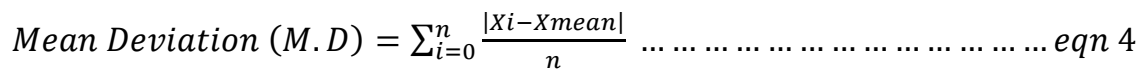

where $\mathrm{X}_{\mathrm{i}}$ is a VO partner's trust level, $\mathrm{X}_{\text {mean }}$ is a mean trust level and $\mathrm{n}$ is the number of VO partners participating. This model considers trust level for each partner as data sets for computing mean trust level for $\mathrm{VO}$, which is denoted as $\mathrm{X}_{\text {mean }}$. Then individual deviation for each VO partner is computed as illustrated in equation 2 and further in equation 3. The mean trust level for all VO partners is computed during the base period.

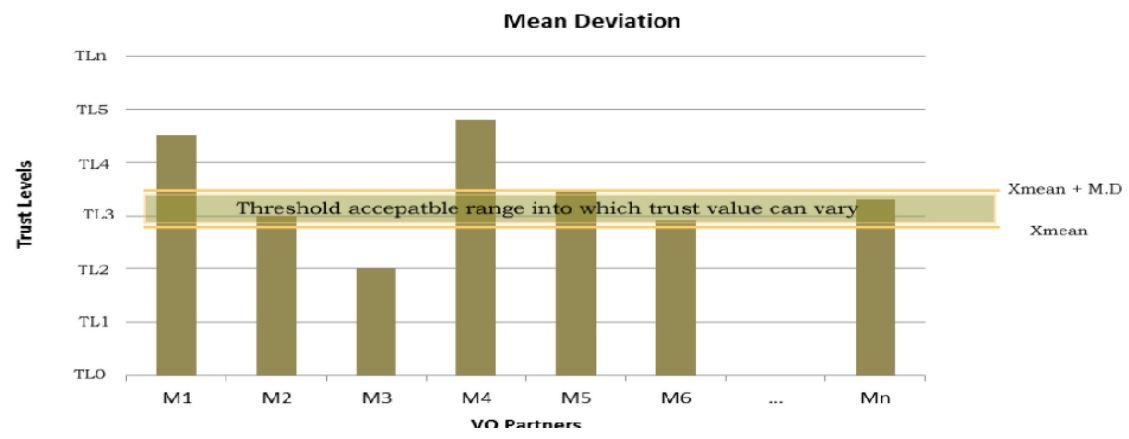

Fig. 3. Trust assessment by mean deviation method

Then VO's mean deviation (M.D) is calculated as illustrated in equation 4. The range Xmean $+M . D$ becomes the acceptable range over which individual VO partner's trust level can oscillate or vary and still that specific partner remain trustworthy (refer figure 4). After a lapse of agreed period (say, semi-annual), each individual VO partner's trust level is assessed and compared to see if it range between and above Xmean +M.D or has fallen belowXmean. If the trust level of a particular VO partner is found to range below $\mathrm{X}_{\text {mean }}$, then such specific partner is said to fail to sustain its trust level and therefore it is less trustworthy.

This is a better model as compared to mean estimation one, because it targets to a range of values (refer figure 3 and figure 4) which is somehow easy to achieve than targeting to a single value which is obviously hard to make. Since deviation is given as absolute value, it is always positive. It does not reflect whether such deviation in trust level was of decrease or increase (figure 3). Though the model creates a customized space for interval limit in trust value for each individual VO partner; the space appears to be biased in one direction. The deviation is an absolute value which is always positive, so that space for interval limit is given by Xmean $+M D$. Its limitations include providing space for trust level limits only in one direction but also it is not standard for all VO partners participating. This approach is very suitable when there is a large set of organizations from which a few need to remain in a VO and thus all those organizations whose trust level has fallen will be first dropped. 


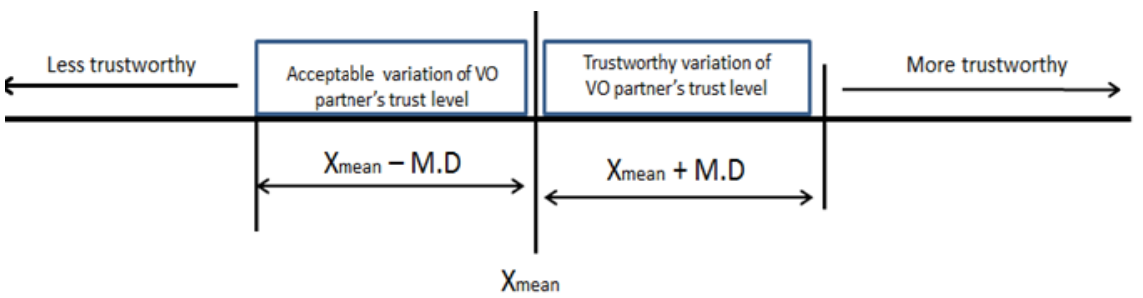

Fig. 4. Linear presentation of VO member's trustworthiness by mean deviation method

\subsection{Third-Level Model: Standard Deviation}

Standard deviation model is a statistical method useful in determining how far is the data spread out in a particular sample or population, and how individual data are close to the mean. This value gives information on how the values of the data set are varying, or deviating, from the mean of the data set. The statistical assessment mechanism which is standard and able to cater for overall weaknesses of both, mean estimation and mean deviation models is a standard deviation model. It requires calculation of trust variation range (Figure 5) based on standard deviation of trust level given by

$$
\text { Standard Deviation }(V s d)= \pm \sqrt[2]{\frac{\sum(\text { square }(\text { Xi-Xmean }))}{N}} \ldots \ldots \ldots \ldots \ldots \text { eqn } 4
$$

Since standard deviation may be positive or negative, then the range for trust value becomes acceptable within the range $\mathrm{V}_{\text {mean }}-\mathrm{V}_{\mathrm{SD}}$ to $\mathrm{V}_{\text {mean }}+\mathrm{V}_{\mathrm{SD}}$. With this model, space interval limits in both directions exists, with their width twice the standard deviation. Since the width is standard, a VO partner becomes equipped with wide space to allow variation in trust level. Such VO partner's individual trust level can vary within its customized acceptable limits evaluated based on its own capacity determined by its current period's trust level to be assessed in the next period. This means, if the VO partner's trust level is within the range, such VO partner has succeeded to strive for its existence and maintained its trust level. If the trust level shoots down below the acceptable minimum trust level, it is said to fail to comply with agreed trust range. The model works by calculating first the mean trust value for all the available VO partners, as

$$
\text { Mean Trust value }(\text { Vmean })=\sum_{i=1}^{n} \frac{V i}{n} \ldots \ldots \ldots \ldots \ldots \ldots \text { eqn } 5
$$

Then, it calculates population variance of the trust values based on mean trust value denoted by $\mathrm{V}_{\text {variance, }}$ as

$$
\text { Population variance }(\text { Vvariance })=\sum_{i=1}^{n} \frac{\text { Square }(\text { Vi-Vmean })}{N} \ldots \ldots \ldots \ldots \text {........eqn } 6
$$

Finally, the model calculates standard deviation for trust level denoted by VSD, as

$$
\text { Standard Deviation in Trust Values }(S D)= \pm \sqrt{\text { Vvariance }} \ldots \ldots \ldots \text {..... eqn } 7
$$




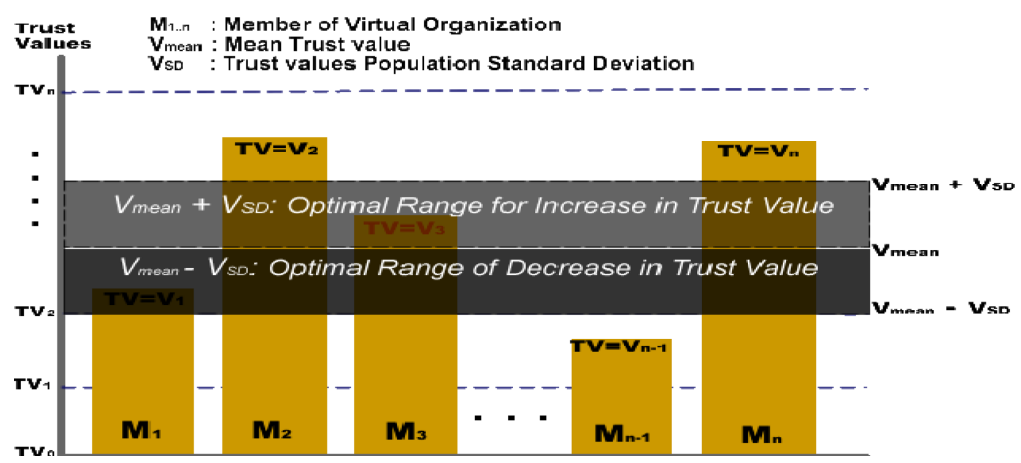

Fig. 5. Model for Trust Sustainability and Evolution Support

Since the standard deviation for trust values may be positive or negative, this implies that it provides an interval of twice standard deviation for which the trust levels variation is allowed. Mathematically, standard deviation measures how widely spread data points are from the mean (refer Figure 6). If more proportion of trust level for the individual VO partners are very close or near to the mean, standard deviation becomes very small number. Since an experiment that yields data with a low standard deviation is said to have high precision, this implies strong bonding among VO partners and VO collaboration is assured. On the other hand, if a high proportion of trust levels lay far from the mean trust level, then the standard deviation is large, and as such the experiment that yields data with a high standard deviation is said to have low precision. This implies creation of gaps in levels of trust, and consequently weakens collaborative bonds. A score below minimum acceptable range signifies that such particular VO partner threatens and weakens bonding factor and hence affects collaboration. Thus, the partner cannot be trusted any more by other partners in achieving common and shared goals. It is important to think about replacing a failed partner by selecting another member from VBE to join the VO. In such environment, the VO is said to evolve.

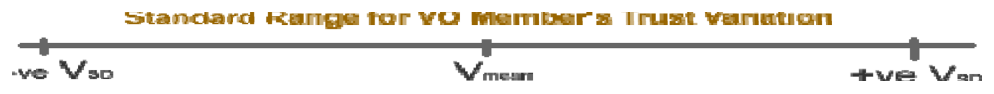

Fig. 6. Range of Trust Values Variation

The implementation of the standard deviation model, employing real case example with real indicators, was done in Trust Sustainability and Evolution (TrustSEv) system; which is not part of this paper.

\section{Conclusion}

This paper has presented models that can be applied to develop approaches for rational trust assessment in VO whose collaboration is already in progress. The VO 
partners need to sustain their levels of trust and undergo evolution if need arises. Since the assessment model for trust assessment during VO formation is insufficient to address trust evolution during $\mathrm{VO}$ operations, then devising new approach is promising. The paper has compared three statistical models namely; mean estimation, mean deviation and standard deviation that each is suitable for different cases. The mean estimation is suitable for dropping partners whose performance in terms of trust level is below the mean.

\section{References}

1. Msanjila, S.S., Afsarmanesh, H.: Trust analysis and assessment in virtual organization breeding environments. I. J. of Production Research ISSN 0020-7543

2. Msanjila, S.S.: On Inter-organizational Trust Engineering in Networked Collaborations: Modeling and Management of Rational Trust, unpublished PhD Theses, University of Amsterdam (2009)

3. Msanjila, S.S., Afsarmanesh, H.: On Modeling Evolution of Trust in Organisations Towards Mediating Collaboration. Production Planning \& Control 22(5-6) (2011)

4. Msanjila, S.S.: Engineering the Evolution of Organizational Trust in Operating Virtual Organization. International Journal of the Academy of Organizational Behavior Management (IJAOBM) 1(2012), 120-138 (2012) ISSN: 1927-565X

5. Wehmeyer, K., Riemer, K.: Trust-building potential of coordination Roles in virtual organizations. The Electronic Journal for Virtual Organizations and Networks 8 (March 2007) 